\title{
Temporary Server for Service Continuity in Next Generation Network
}

\author{
Rajina R. Mohamed
}

\begin{abstract}
Real-time applicationisone of the internet-based applications that function with in a time frame that require a quick response between client and server. It has been improved to fulfill user's demand towards Next Generation Network(NGN), by injecting intensive and media-rich elements in the applications. All these innovations need sufficient bandwidth to ensure them to be delivered continuously, smoothly and successfully to the end users. However, current scenario has seen the internet population has raised rapidly so does the potential size of its traffic hat give significant impact to the online service. Hence, an efficient infrastructure is required to satisfy the demand of internet applications such as multimedia streaming. In this paper, we describe a frame work to allocate a temporary server to serve local clients within the same network domain during high traffic. This proposal has been tested on video streaming application to see the continuity of the service.
\end{abstract}

Index Terms-Temporary server, secondary server, primary server, service continuity, next generation network.

\section{INTRODUCTION}

Nowadays, the popularity of internet application has brought to the proliferation of internet used. The variety of media social application for instances Facebook, Twitter and Instagram as well the evolution of mobile phone resulting to the exponential of internet traffic in Next Generation Network (NGN) [1] today. As it is portable and handy, it is becoming a medium for various businesses purposes for anytime and anywhere. Likewise, the demand for internet services is increasing and not solely limited to static applications, but demand for dynamic content applications has grown up exponentially.

As for the real-time dynamic content (e.g.real-time media streaming) [2], it requires immediate response from the server so as the service is continuously supplied. However, as the internet grows, so does the potential size of the traffic and the impact is significant to a real-time application.The real-time application must be functioned within a time frame that the user senses directly. In addition, the latency must be less than the defined value, usually measured in a quick second [3]. However, the satisfactory rate of delivery media streaming to the bandwidth offered by the network is not always able to meet the demand [4]-[6]. As a consequence, an adaptive approach should be considered in order to handle the exponential of internet users, and to fulfill the demands towards the real-time applications.

In order to satisfy such target, we proposed a solution to reduce and distribute traffic by diverting client's request

Manuscript received April 4, 2014; revised August 18, 2012.

Rajina R. Mohamed is with the UNITEN, Kuala Lumpur, Malaysia (e-mail: rajina@uniten.edu.my). from the primary server to the local alternative server instead of only pointing to one source of server. This approach is by remotely allocates a temporary server at local area that is near to the client domain network. This temporary server is allocated during network high congestion and it is done by primary server at a runtime.

The temporary local server will serve the group of client in the same domain network. This method is diverse to the previous approaches such as Peer-to-Peer (P2P) networking, Content Delivery Network (CDN) and Replication/cache server [7]-[9]. The previous solutions are not practical due to high cost to allocate the physical server, high maintenance and also need to verify the accurate location of the servers. In contrast, our proposal is more cost saving as we need only some space temporary during the network congestion and the server will return back to the primary server once the task of serving client's request have accomplished.

\section{PROBlem SCENARIO AND SOLUTIONS}

Fig. 1 shows a scenario of the current problem in public network. All clients are pointing to primary server that located at public network. Network traffic becomes more congested whenever the numbers of clients increase. Due to this scenario, it will reduce the efficiency of internet service offered.

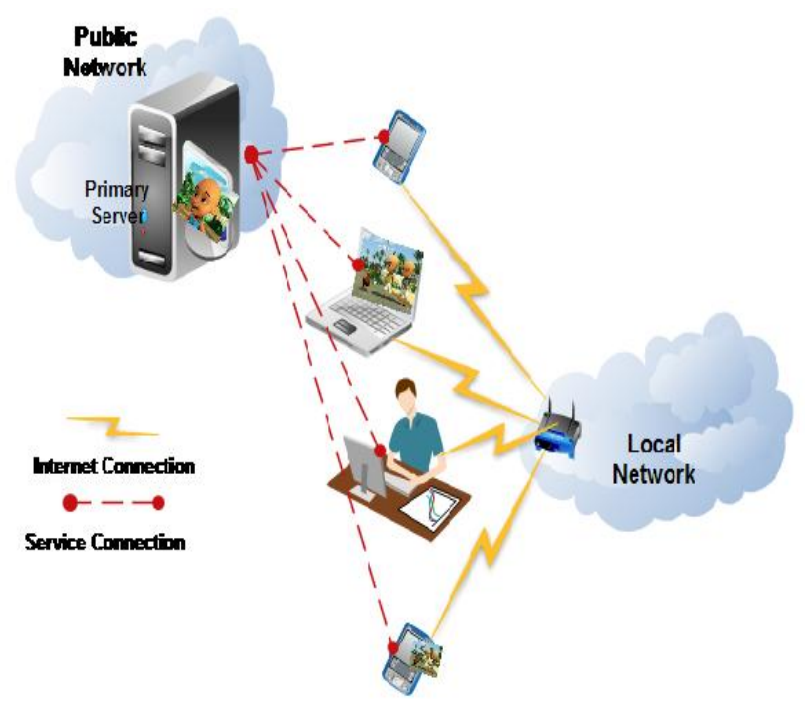

Fig. 1. Problem scenario.

This problem will be solved by reducing the direct connection from clients to the origin content server (Primary Server) which is resides at public network, as shown in Fig. 2.

Based on some threshold value, clients will be redirected 
to the temporary server allocated which resided at local network nearby the clients during network congestion.

Secondary Server will act as server local clients in the same network domain instead of pointing all to the Primary Server. This secondary server will serve local clients as good as served by the Primary Server. This will increase the efficiency of internet service by avoiding network traffic congestion during peak time. If the primary server found traffic at that time had been reduced, then the primary server will call back the local client or a local client will be removed from this time, and the primary server will act as a streaming server.

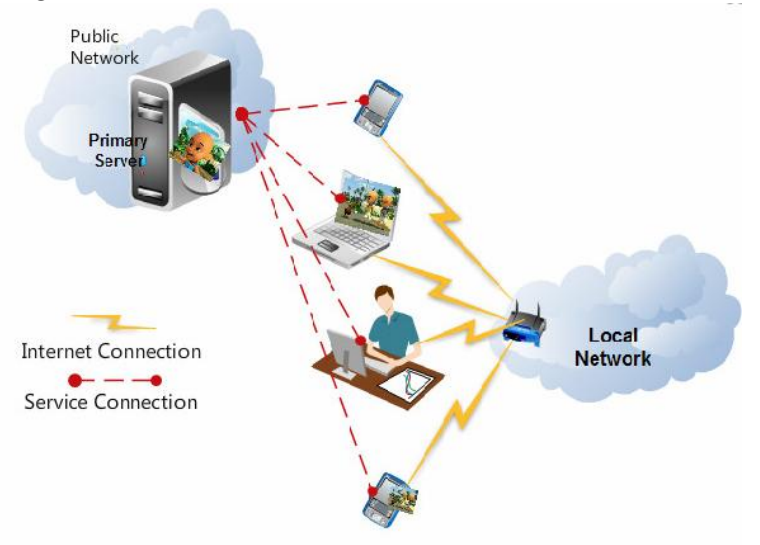

Fig. 2. Proposal solution.

\section{PROPOSAL IMPLEMENTATIONS}

\section{A. Proposal Architecture}

Our proposal is divided into four main components: Primary Server, Secondary Server, Traffic Manager and Clients. Fig. 3 shows the main components of the proposal.

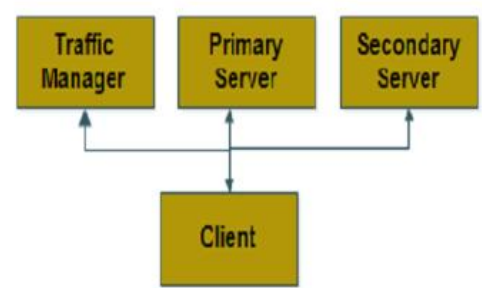

Fig. 3. Proposal architecture.

Primary Server acts as a source of contents that is requested by clients, resided at public network. Secondary Server is a temporary content provider resided at local area network that will serve local clients during high traffic. Traffic Manager is a component that is to get all information of request-response activity between clients and server as well as to record all clients' information. Traffic Manager will update all connected and disconnected clients either to the public network or local network. The redirection process from Primary Server to Secondary Server is decided by Traffic Manager. In this paper, the decision to redirect to the Secondary Server is done by Traffic Manager by calculate the number of clients. The redirection must obey the condition by (1), as in:

$$
\sum_{n=1}^{m} C l_{n}>C l_{t} \text { then } S_{1} \rightarrow S_{2}
$$

where $C l_{n}=n, n$ is number of client, $m$ is the maximum number of client, $t$ is the defined threshold, $S_{1}$, is Primary Serverand $S_{2}$ is Secondary Server. If number of client, $C l_{n}$ is more than defined threshold value, $C l_{t}$, then Traffic Manager will trigger the Secondary Server to take place of streaming the video. Secondary Server wills response back to the Traffic Manager with its streaming address. The streaming address of Secondary Server will be informed to all clients in the same network group so as clients will request the service from Secondary Server. Consequently, the connection to the Public Server will be terminated.

\section{B. System Flowchart}

Our system proposal is further explained by the flowchart in Fig. 4. The prototype is started with all clients request for streaming to the Primary Server, and throughout the time Traffic Manager will capture the IP address of all clients, their gateway as well as the address of streaming server. All such information is important in order to divert the stream to local client when necessary. Traffic Manager will also count the number of clients that request a service from Public Server until it exceeds the threshold value. When the number of clients detected exceeds the threshold value, Traffic Manager will notify Secondary Server to take place to serve the local client. Next, Traffic Manager will notify the client about the address of the Secondary Server. After receiving notification from the Traffic Manager of the new address that is closer to them, then clients will begin to redirect the request to the local server now clients are being connected to the Secondary Server, while continue watching the video. Consequently, the clients' connection to the Primary Server will be terminated.

\section{Proposal Testbed}

A testbed for our proposal has been setup to see the impact of temporary secondary server to the continuity of service during high traffic. The end target of this experiment is to see the continuity of the service among clients when they had been connected to the temporary secondary server without any service interruption. Basically, the server redirection process is transparent to the clients.

In order to see the seamless service, we have tested it on online video streaming application as video streaming application is more delay sensitive and it requires continuously streaming delivery throughout the session. Furthermore, the video streaming session can be perceived by the end users. We make use VLCJ [10] in order to provide a client-server video streaming. The VLCJ is an open-source, code was customized in order to fulfill our target.

Three laptops with Ubuntu pre-installed were used in this experiment. They were function as Primary Server, Traffic Manager, Secondary Server as well as clients. Primary Server and Secondary Server were installed with VLC Media Player as a streaming server that can act to serve video streaming for clients.Both Primary and Secondary Server were setting with IPv4 address, 192.168.0.103 and 192.168.0.101 respectively. One laptop was programmed as Traffic Manager to manage all clients' activities and also to measure network traffic. There are three clients involved in 
this experiment named as Client1, Client2 and Client3. However, to simplify the connection process for the proofof-concept, all the three laptops were also used as clients throughout the time. Fig. 5 shows the testbed of the proposal while Fig. 6 depicts the actual setup of the testbed in our lab.

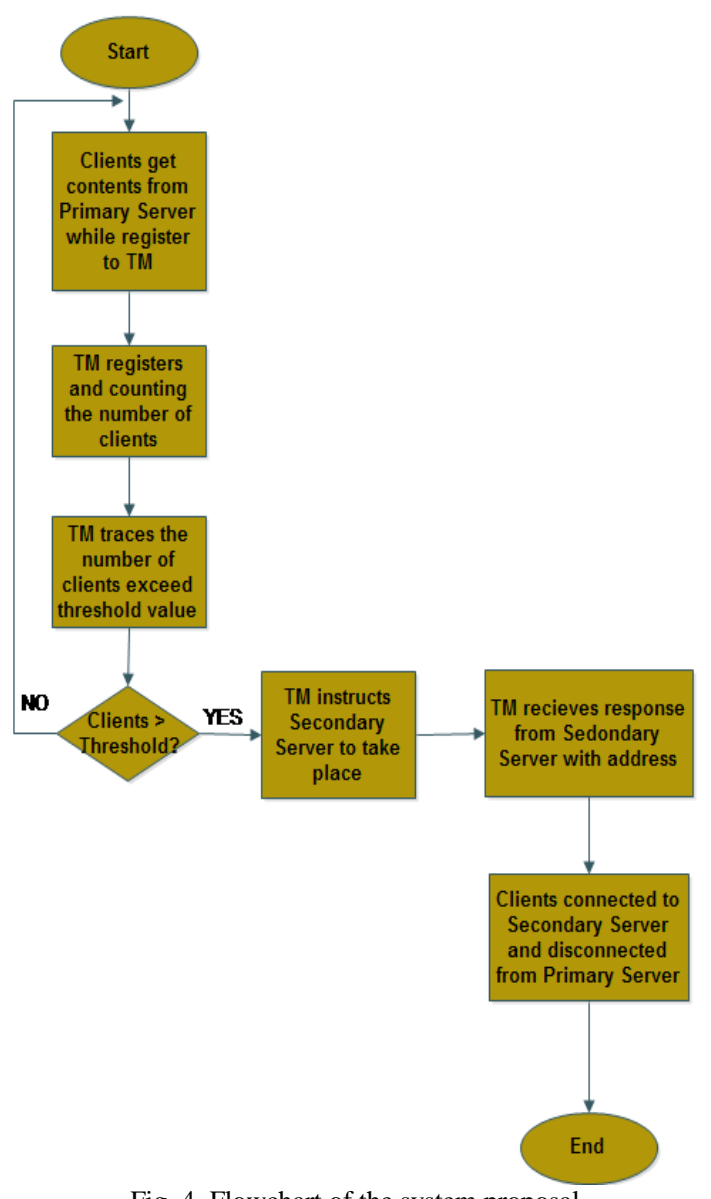

Fig. 4. Flowchart of the system proposal.

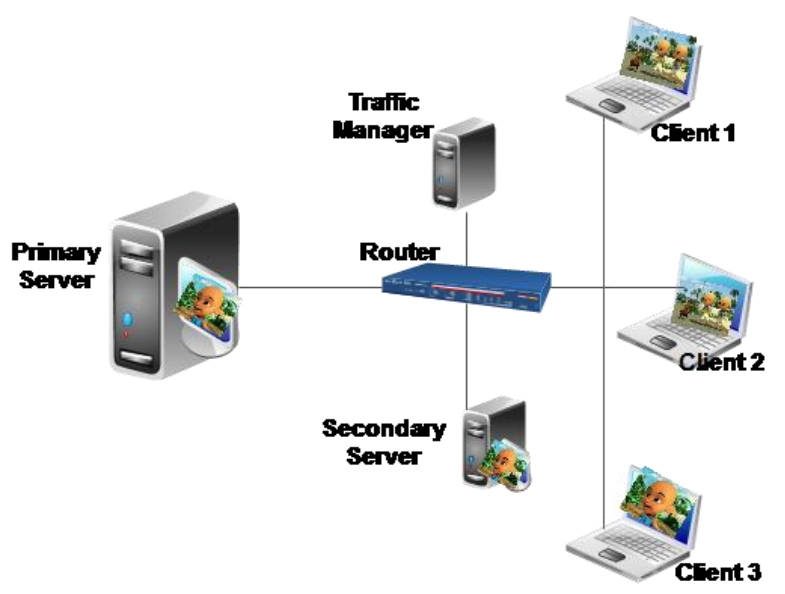

Fig. 5. Proposal testbed setup.

\section{Proposal Testbed Plan and Result}

All components architecture as shown in Fig. 5 will communicate to each other to realize the streaming service. Test plan scenario will take place as follows:

- Run Primary Server to stream the video contents.

- Run Secondary Server and Traffic Manager.

- Run Client 1 and Client 2 simultaneously, to get streaming content from Primary Server.
- After some point, run Client 3 to see the continuity of the service once the content server is hand off from Primary Server to the Secondary Server.

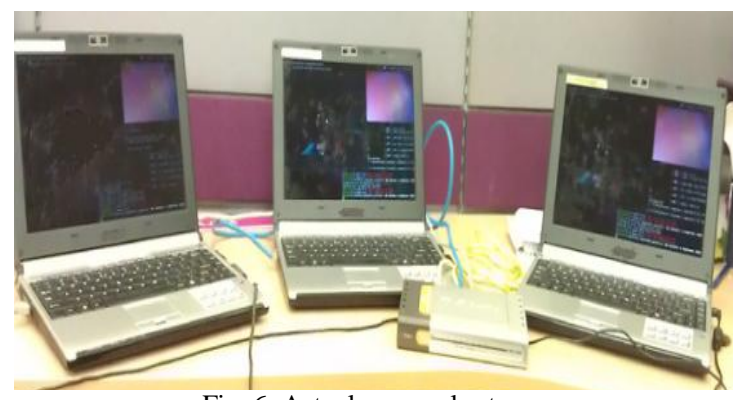

Fig. 6. Actual proposal setup.

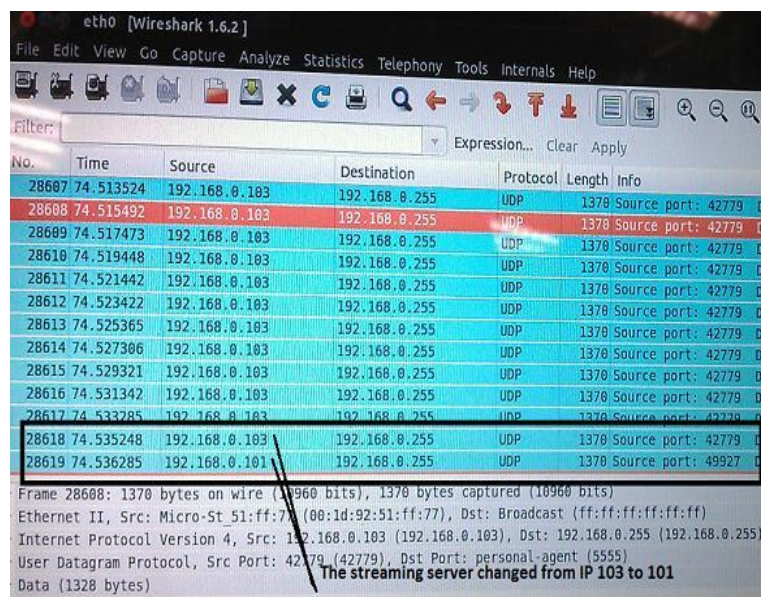

Fig. 7. Wireshark- the changing of server between primary and secondary server.

The system has been run and executed more than 15 times to get the consistence result. Fig. 7 shows the result captured by Wireshark application [11]. The result shows that the source of UDP session has changed from 192.168.0.103 to 192.168.0.101. For the testing purpose, we ask a few users to experience the quality of streaming before and after the handover before and after traffic congestion. During the server handover from 192.168.0.103 (Primary Server) to 192.168.0.101 (Secondary Server), user did not facing any delay or video distortion. From the user perspective, the session has run smooth and seamless without any interruption. Besides, users did not facing any video lagging or content delay while the experiment was conducted.

\section{CONCLUSION}

We have proposed a method to reduce network traffic for the purpose of improving the efficient of internet service, especially during high congestion. The proposed system has been tested on a video stream to see the effectiveness of online service continuity. Result shows the current online session continues uninterrupted and without delays. This shows that the proposal can be applied to an online system such as online assessment and also any other internet service that require quick request-response. This is an initial study for this proposal and the objective of the experiment has been achieved. To enhance the current study, we will conduct simulation as well as experimental study that will cover more clients and various domain sub network. 


\section{REFERENCES}

[1] A. Basili and E. Paglia, "NGNs: A plat form enhancing technological multimedia services in cultural heritage innovations," in Proc. 20 IEEE International Workshops on Enabling Technologies (WETICE), 2011, pp. 268-273, 2011.

[2] R. M. Rajina, "A Study of information server over seamless service continuity during network handover," in Proc. INNOV2012, IARIA Press, pp. 37-41, 2012 .

[3] R. Karrer and T. Gross, Dynamic Had off of Multimedia Streams, ACM Press, 2011.

[4] S. Devillechaise et al., "Dynamic adaptation of the squid web cache with Archne," Software IEEE, vol. 23, Jan. 2006, pp. 34-41.

[5] A. Vakali, "Proxy cache replacement algorithms: ahistory-based approach," World Wide Web, 2001, vol. 4, pp. 277-297.

[6] D. Cicco, "An experimental evaluation of akamai adaptive video streaming over HSDPA network," in Proc. IEEE International Symposium on Computer-Aided Control System Design, pp. 13-18, 2011.

[7] S. Xu and H. Shen. Qos-Oriented Content Delivery in E-Learning System. [Online]. Available: http://ieeexplore.ieee.org/stamp/stamp.jsp?arnumber=06316570
[8] CDN Reviews. Advantages and Disadvantages of Using a Content Delivery Network. [Online]. Available: http://www.cdnreviews.com/cdn-advantages-disadvantages/.

[9] S. I. Lee, D. M. Lee, and S. G. Kang, "Challenges to P2Plive video streaming over mobile heterogenous network," in Proc. IEEE Computer Science and Convergence Information Technology (ICCIT), 2011, pp. 36-41.

[10] VLCJ. Homepage-Caprica Software. [Online]. Available: http://www.capricasoftware.co.uk/vlcj/.

[11] Wireshark. [Online]. Available: http://www.wireshark.org.

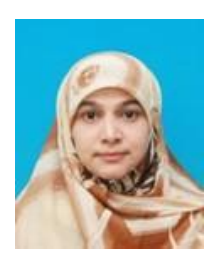

Rajina R. Mohamed received her bachelor degree in computer science from University of Putra (UPM), Malaysia in 1995. She then pursued her MSc in computer science majoring in distributed computing at the same university (UPM), and completed her MSc in 2003. She is currently works as a lecturer in UNITEN, Kuala Lumpur, Malaysia since September 2013. Previously, she was a researcher at MIMOS Berhad focusing on computer networking. She is a researcher in MIMOS Berhad since 1996 involved with various field of research includes network information security, wireless communication (IPv4andIPv6), digital home and e-learning. rajina has published several publications and filed several patents on her research findings. She is a member of IEEE. 